\title{
Superoxide Dismutase-A Genetic Marker in Cardiomyopathies
}

\author{
S.D. Annapurna ${ }^{1}$, B. Ushasree ${ }^{1}$, T.R. Reena ${ }^{1}$, C. Narasimhan ${ }^{2}$, B. SomaRaju ${ }^{2}$ and N. Pratibha ${ }^{1}$
}

\author{
1. Department of Genetics, Osmania University, Hyderabad 500 007, Andhra Pradesh, India \\ E-mail: anusha_annu@hotmail.com
}

2. Consultant Cardiologists, CARE Hospital, Nampally, Hyderabad 500 001, Andhra Pradesh, India

\begin{abstract}
KEY WORDS Dilated cardiomyopathy; hypertrophic cardiomyopathy; phenotypes; genetic heterogeneity; risk prediction
\end{abstract}

\begin{abstract}
Cardiomyopathies are a heterogeneous group of heart muscle disorders responsible for a great deal of morbidity and mortality. Dilated, hypertrophic and restrictive are the three major categories of cardiomyopathies. Since reactive oxygen species has been implicated in wide range of genetic disorders and the role of antioxidant like superoxide dismutase as a scavenger has been highlighted, the present study envisages on identifying the specific electromorphic association of superoxide dismutase with cardiomyopathies and to delineate the genetic heterogeneity based on specific alleles at risk. Phenotyping was carried out on $8 \%$ PAGE of the red cell membrane samples following Davies (1964) and Beauchamp's (1975) protocols. Blood samples from 62 dilated cardiomyopathy, 80 hypertrophic cardiomyopathy and 86 healthy individuals were collected from CARE Hospitals and voluntary blood donor camps, Hyderabad. Significant association of homozygous SOD A*1 $\left(\chi^{2}=7.58\right)$ alleles with dilated cardiomyopathy and heterozygous SOD A*2-1 $\left(\chi^{2}=5.89\right)$ alleles with hypertrophic cardiomyopathy was observed, resulting in significant deviation of allelic frequency in cardiomyopathy group compared to the control group highlighting that individuals carrying SOD A*1 $\left(\chi^{2}=7.588\right)$ and SOD A*2-1 $\left(\chi^{2}=5.89\right)$ alleles may be at a greater risk for dilated and hypertrophic cardiomyopathy respectively. Thus SOD as a genetic marker may help in risk prediction and in delineating genetic heterogeneity of the condition and the role of superoxide dismutase with specific electromorphic association in influencing endogenous nitric oxide production and energy pathways by altering the structure and function of the cell membranes is discussed.
\end{abstract}

\section{INTRODUCTION}

Cardiomyopathies are defined as primary disorders of cardiac muscle and include three specific types (a) dilated cardiomyopathy (DCM) (b) hypertrophic cardiomyopathy (HCM) and (c) re-

Address for correspondence: Dr. Pratibha Nallari, Associate Professor, Department of Genetics, College of Science, Osmania University, Jamai Osmania Post office, Hyderabad 500 007, Andhra Pradesh, India.

Phone: 040-56540482, 040-27830242,

E-mail: prathinallari@yahoo.com strictive cardiomyopathy (RCM).

All forms of cardiomyopathy may be inherited or acquired, but in either circumstance, the underlying cause is typically unknown. Among these, dilated and hypertrophic cardiomyopathies are clinically and genetically heterogeneous disorders. Hypertrophic cardiomyopathy is characterized by an increased left ventricular mass or an increase in the mass of interventricular septum in the absence of a secondary cause, while in dilated cardiomyopathy the heart is enlarged and functions poorly with both the left and right sides of the heart involved, resulting in heart weakening and inefficient pumping of blood around the body as cited by Davies (1984).

Fridovich (1975) reported that SOD, a genetic marker acts as an antioxidant and catalyses the dismutation of oxygen radicals to yield hydrogen peroxide and oxygen, which has been implicated as being essential in defense against the potential toxicity of oxygen. Two identifiable isoenzymes SOD A, a cytoplasmic, soluble, $\mathrm{Cu}-$ $\mathrm{Zn}$ containing enzyme and SOD B, a mitochondrial, insoluble, Mn containing enzyme is encoded by genes localized on 6q25.3 and 21q22.1 chromosomes respectively as revealed by Del Villano et al. (1979).

DeCroo et al. (1988) reported the polymorphism with respect to SOD A with three phenotypes identified as SOD A*1, SOD A*2-1, SOD $\mathrm{A}^{*} 2$ encoded by two allelic forms SOD A*1 and SOD A*2 respectively and inherited as co-dominant alleles. Since no information with regards to SOD and its electromorph association, if any, is available in cardiomyopathies, such a study is imperative which may help in risk prediction and in delineation of genetic heterogeneity of the condition.

Therefore, the present study aims at identifying specific electromorph association of SOD $\mathrm{A}$ and its role in the etiology of cardiomyopathies. 


\section{MATERIALS AND METHODS}

Blood samples from 62 dilated cardiomyopathy, 80 hypertrophic cardiomyopathy patients and 86 healthy controls matched for age and sex, has been collected from CARE Hospitals, Hyderabad and voluntary blood donor camps, Hyderabad. $5 \mathrm{ml}$ of venous blood was collected from each individual in a vacutainer containing EDTA. The samples were centrifuged at $2000 \mathrm{rpm}$ for about 10 min till a clear supernatant was obtained and stored in eppendorff tubes in the refrigerated condition until further use.

The whole blood was washed with normal saline thrice and then centrifuged with ice-cold water to obtain red cell membranes for the analysis of superoxide dismutase phenotypes.

Apart from clinical data and blood samples, relevant epidemiological information like age, age at onset, symptoms, familial status, consanguinity and pedigree information extending over 2-3 generations was also obtained from each individual on personal interrogation.

An 8\%, 1.0 mm PAGE gel was prepared taking $1 \mathrm{ml}$ Tris (hydroxy methyl) methylamine solution, $2 \mathrm{ml}$ Acryl amide/ bis acryl amide solution, $4 \mathrm{ml}$ of Ammonium per sulfate solution and $1 \mathrm{ml}$ of distilled water following Davies protocol (1964). After polymerization of the separating gel, a 5\% stacking gel solution was prepared in the ratio of 1:2:4:4 with $1 \mathrm{ml}$ tris, $2 \mathrm{ml}$ acryl amide/ bis acryl amide, $4 \mathrm{ml}$ ammonium per sulfate and $4 \mathrm{ml}$ distilled water and poured over the $8 \%$ separating gel and allowed to polymerize after inserting a sample comb. Later the plates were fixed to the vertical slab gel electrophoretic unit and $0.05 \mathrm{M}$ tris glycine stock buffer was diluted 10 folds and was poured in upper and lower tanks, which were later, connected to the anodic and cathodic ends of the DC power supply.

Later the sample was prepared by adding a drop of bromophenol blue indicator to 40ul of red cell membrane mixed thoroughly and loaded on to the sample slot of the gel. The electro- phoresis was carried out at $4^{\circ} \mathrm{C}$ for 2 hours at $20 \mathrm{~mA}$ constant current. The run was carried out till the indicator migrated to the other end of the gel as a blue sharp band. The gel was removed and placed in a tray and incubated in freshly prepared staining solution which consisted of $5 \mathrm{ml}$ of phosphate buffer ( $\mathrm{pH} 7.8$ ), $8.5 \mathrm{ml}$ of riboflavin, $10 \mathrm{mg}$ of nitroblue tetrazolium chloride and $220 \mu \mathrm{l}$ of TEMED in $50 \mathrm{ml}$ distilled water. Later, the gel was washed and placed in distilled water and exposed under a bright fluorescent light till the background turned blue and clear achromatic bands appeared as cited by Beauchamp and Fridovich (1971). Based on their mobility in the gel, the phenotypes of SOD A were identified as 2-2, 2-1 and 1-1, respectively.

Allelic frequencies were estimated using Hardy Weinberg equilibrium and $\chi^{2}$ test of analysis ( $2 \times 2$ contingency) and Woolf's test of association was carried out to interpret the results statistically as cited by Emery (1984).

\section{RESULTS}

Table 1 presents the frequency distribution of superoxide dismutase phenotypes in normal subjects and cardiomyopathic patients. The distribution of superoxide dismutase phenotypes in the normal individuals were $76 \%$ of $2-2,13 \%$ of 2-1 and $12 \%$ of 1-1 phenotypes, while in dilated cardiomyopathy $56.5 \%$ were of $2-2,21 \%$ of $2-1$ and $22.6 \%$ of 1-1 phenotypes and in hypertrophic cardiomyopathy $64 \%$ were of $2-2,29 \%$ of $2-1$, $7.5 \%$ of $1-1$ phenotypes. An increased frequency of SOD A*1 (22.6\%) and decreased frequency of SODA*2 $(56.45 \%)$ phenotypes were observed in dilated cardiomyopathic patients while SODA $* 2-1$ phenotype was found to be preponderant in hypertrophic cardiomyopathy $(29.0 \%)$ compared to the control group.

The test of association of superoxide dismutase phenotypes with the disease condition compared to the control group is presented in table 2. An increased predisposition

Table 1: Frequency distribution of SOD phenotypes in control and disease groups

\begin{tabular}{|c|c|c|c|c|c|c|c|}
\hline & \multicolumn{6}{|c|}{ SOD Phenotypes } & \multirow[b]{3}{*}{ Total } \\
\hline & \multicolumn{2}{|c|}{$S O D A * 2-2$} & \multicolumn{2}{|c|}{$S O D A * 2-1$} & \multicolumn{2}{|c|}{ SODA* 1-1 } & \\
\hline & $n$ & $\%$ & $n$ & $\%$ & $n$ & $\%$ & \\
\hline Control & 65 & 76.0 & 11 & 13.0 & 10 & 12.0 & 86 \\
\hline Dilated Cardiomyopathy & 35 & 56.45 & 13 & 21.0 & 14 & 22.6 & 62 \\
\hline Hypertrophic Cardiomyopathy & 51 & 64.0 & 23 & 29.0 & 6 & 7.5 & 80 \\
\hline
\end{tabular}


of homozygous SOD A*1 phenotypic individuals to dilated cardiomyopathy $\left(\chi^{2}=7.558\right)$ was observed while a significant preponderance of heterozygous SOD A*2-1 phenotypic individuals $\left(\chi^{2}=5.89\right)$ to hypertrophic cardiomyopathy were noted.

Relative risk estimates of superoxide dismutase phenotypes in disease and control group is presented in table 3. A significant association of SOD A*1 phenotypes with dilated cardiomyopathy $\left(\chi^{2}=6.028\right)$ and SOD A*2-1 with hypertrophic cardiomyopathy $\left(\chi^{2}=5.750\right)$ was observed.

Table 4a gives the allelic frequency of SOD
$\mathrm{A} * 1$ and SOD A*2 alleles in disease and control group. The allelic frequency of SOD A*1 was reported to be 0.181 in control, 0.34 in dilated cardiomyopathy and 0.219 in hypertrophic cardiomyopathy while SODA*2 was found to be 0.819 in control, 0.66 in dilated cardiomyopathy and 0.781 in hypertrophic cardiomyopathy, thus indicating a decrease in allelic frequency of SOD $A * 2-1$ and an increase in allelic frequency of SOD A*1 in both dilated and hypertrophic cardiomyopathy in comparison to the control group.

Table $4 \mathrm{~b}$ gives the genotypic frequency distribution and test for Hardy Weinberg equilibrium of superoxide dismutase in control and dis-

Table 2: Test of association of SOD phenotypes with the disease in comparison to the control groups:

\begin{tabular}{|c|c|c|c|c|c|}
\hline \multirow{2}{*}{$\begin{array}{c}\text { SOD } \\
\text { Phenotypes }\end{array}$} & \multirow[b]{2}{*}{ Control } & \multicolumn{2}{|c|}{ Cardiomyopathy } & \multicolumn{2}{|c|}{$\chi^{2}$ values } \\
\hline & & Dilated & Hypertrophic & $D C M$ & $H C M$ \\
\hline $2-2$ & 65 & 35 & 51 & - & - \\
\hline $2-2$ vs $2-1$ & 11 & 13 & 23 & 2.996 & $5.89 *$ \\
\hline $2-2$ vs $1-1$ & 10 & 14 & 6 & $7.558 * *$ & 5.239 \\
\hline $2-2$ vs $2-1 / 1-1$ & 21 & 27 & 29 & $6.063 * *$ & 2.750 \\
\hline $2-2$ vs $1-1$ & 10 & 14 & 6 & 0.084 & $4.05 *$ \\
\hline
\end{tabular}

RR-Relative Risk; *p $<0.05, * * \mathrm{p}<0.01$

Table 3: Relative risk estimates of SOD phenotypes in disease and control groups

\begin{tabular}{|c|c|c|c|c|c|c|c|}
\hline \multirow{3}{*}{$\begin{array}{c}\text { SOD } \\
\text { Phenotypes }\end{array}$} & \multirow{3}{*}{$\begin{array}{c}\text { Control } \\
n\end{array}$} & \multicolumn{6}{|c|}{ Cardiomyopathy } \\
\hline & & \multicolumn{3}{|c|}{ Dilated } & \multicolumn{3}{|c|}{ Hypertrophic } \\
\hline & & $n$ & $R R$ & $\chi^{2}$ & $n$ & $R R$ & $\chi^{2}$ \\
\hline $2-2$ & 65 & 35 & - & - & 51 & - & - \\
\hline $2-2$ vs $2-1$ & 11 & 13 & 2.194 & 2.955 & 23 & 2.66 & $8.750 * *$ \\
\hline $2-2$ vs $1-1$ & 10 & 14 & 2.600 & $4.260 *$ & 6 & 0.764 & 0.239 \\
\hline $2-2$ vs $2-1 / 1-1$ & 21 & 27 & 2.400 & 6.028 & 29 & 1.76 & 2.775 \\
\hline $2-2$ vs $1-1$ & 10 & 14 & 1.184 & 0.084 & 6 & 0.286 & $3.903^{*}$ \\
\hline
\end{tabular}

Table 4a: Allelic frequenc distribution of SODA* 1 and SOD $A * 2$ in control and disease groups

\begin{tabular}{cccccccc}
\hline \multirow{2}{*}{ Alleles } & Control & \multicolumn{2}{c}{ Cardiomyopathy } & & \multicolumn{3}{c}{$\chi^{2}$ Values } \\
\cline { 3 - 4 } \cline { 7 - 8 } & & Dilated & Hypertrophic & & Control & DCM & $H C M$ \\
\hline 2 & 0.819 & 0.66 & 0.781 & & $19.238^{* *}$ & $8.904^{* *}$ & 1.329 \\
1 & 0.181 & 0.34 & 0.219 & & & & \\
\hline
\end{tabular}

Table 4b: Genotypes frequency distribution in disease and control groups and test for Hardy-Weinberg equilibrium

\begin{tabular}{|c|c|c|c|c|c|c|c|c|c|}
\hline \multirow{3}{*}{$\begin{array}{c}\text { SOD } \\
\text { Phenotypes }\end{array}$} & & & & \multicolumn{6}{|c|}{ Cardiomyopathy } \\
\hline & \multicolumn{3}{|c|}{ Control } & \multicolumn{3}{|c|}{ Dilated } & \multicolumn{3}{|c|}{ Hypertrophic } \\
\hline & Obs & $\operatorname{Exp}$ & $\chi^{2}$ & Obs & Exp & $\chi^{2}$ & Obs & $\operatorname{Exp}$ & $\chi^{2}$ \\
\hline $2-2$ & 65 & 57.68 & 0.928 & 35 & 27.0 & 2.370 & 51 & 48.79 & 0.100 \\
\hline $2-1$ & 11 & 25.49 & $8.236 * *$ & 13 & 27.82 & $7.894 * *$ & 23 & 27.36 & 0.694 \\
\hline $1-1$ & 10 & 2.817 & $18.31 * *$ & 14 & 7.16 & $6.534 * *$ & 6 & 3.83 & 1.229 \\
\hline Total & 86 & - & - & 62 & - & - & 80 & - & - \\
\hline
\end{tabular}


ease group. A significant deviation from the Hardy-Weinberg equilibrium was observed in dilated cardiomyopathic cases $\left(\chi^{2}=7.894\right)$ and control groups $\left(\chi^{2}=18.31\right)$ while no such deviation was found in hypertrophic cardiomyopathy.

\section{DISCUSSION}

Superoxide dismutase A is a peroxisomal cytosolic enzyme widely distributed in the cell cytosol and in the cell nucleus. Studies by Zimmerman et al. (1973) revealed that superoxide dismutase protects against lipid peroxidative muscle damage. It may be suggested that zincdeficient SOD may participate in both sporadic and familial form of the condition by an oxidative mechanism involving nitric oxide endogenous production. Inhibition of SOD is also known to cause accumulation of cellular superoxide radical and leads to free radical-mediated damage to mitochondrial and red cell membranes with the release of cytochrome c.

Since reactive oxygen species (ROS) have been implicated in a wide range of degenerative processes including amyotrophic lateral sclerosis, ischemic heart disease, Alzheimer disease, Parkinson disease, and aging as indicated by $\mathrm{Li}$ et al. (1995). ROS generated by mitochondria as the toxic by-products of oxidative phosphorylation, may influence the energy-generating pathways. Earlier studies by Huang et al. (2000) reported that inactivation of the mitochondrial form of SOD in mice may result in dilated cardiomyopathy, hepatic lipid accumulation, and early neonatal death, hence polymorphic variation of SOD A in cardiomyopathies was examined.

Studies by Nishi et al. (1995) and Hiroi et al. (1999) also revealed that the genetic risk factors associated with nonfamilial idiopathic cardiomyopathy (IDC) in Japanese, wherein polymorphisms in the SOD2 and HLA-DRB1 genes indicated a significant excess of homozygotes for the $\mathrm{V}$ allele (the presence of valine vs alanine (A type) in the leader peptide of SOD2 at position 16 and an increase in the frequency of the HLADRP1*1401 allele in patients with IDC suggesting that these 2 genetic markers (SOD2-VV genotype and DRB1*1401) may play a synergistic role in controlling the susceptibility to nonfamilial IDC.

Further studies by Novak et al. (1980) suggested that the locus might be 'regulatory' in nature both for cytosolic and mitochondrial form of SOD, hence altered structure and function of cell membranes may result which may have a key pathogenetic role in this particular condition.

Alternatively, Lin et al. (1997) suggested in view of the antioxidant functions involved in the ischemic injury, the expression of endogenous antioxidant enzymes in the tissues with and without HCM, showed a significant increase of $\mathrm{Cu}$, $\mathrm{Zn}$-superoxide dismutase (SOD), and decrease of catalase (CAT) activities in the various areas of HCM hearts.

It was demonstrated that SOD/CAT ratios in the HCM hearts were also found to be significantly higher than in normal hearts, which can be dramatically correlated to the severity of cardiac hypertrophy. The altered SOD/CAT ratio may also be consistent with increase in lipid damage, thus hypothesizing that the elevated SOD combined with an inadequate amount of $\mathrm{H} 2 \mathrm{O} 2$ scavenging enzyme may lead to HCM heart at oxidative stress risk.

In conclusion the study highlights a significant risk of homozygous SOD A*1 phenotypic individuals to dilated cardiomyopathy and heterozygous SOD A* 2-1 phenotypic individuals to hypertrophic cardiomyopathy. Thus superoxide dismutase could help in delineating the genetic heterogeneity of cardiomyopathies with varying association of superoxide dismutase alleles with the type of cardiomyopathy and can be considered as a genetic marker in genetic risk prediction.

\section{ACKNOWLEDGEMENTS}

Authors are grateful to the Department of Biotechnology (DBT), New Delhi for providing financial assistance to carry out the work in Cardiomyopathies.

\section{REFERENCES}

Beauchamp C, Fridovich I 1971. Superoxide dismutase: Improved assays and an assay applicable to acrylamide gels. Analyt Biochem, 44: 276-287.

Davies BJ 1964. Disc electrophoresis II. Method and application to human serum proteins. Ann N Y Acad Sci, 121: 404-464.

Davies MJ 1984. The current status of myocardial disarray in hypertrophic cardiomyopathy. Br Heart $J$, 51: 361-366.

DeCroo S, Kamboh MI, Leppert M, Ferrell RE 1988. Isoelectric focusing of superoxide dismutase. Report of the unique SOD $\mathrm{A}^{*} 2$ allele in a US white 
population. Hum Hered, 38: 1-7.

Del Villano BC, Tischfield JA, Schacter LP, Stilwil D, Miller SI 1979. Cupro-zinc superoxide dismutase. A possible biologic marker for alcoholism (studies in black patients). Alcohol Clin Exp Res, 3: 291296.

Emery AEH 1984. Statistical Methods in Medical Genetics. UK: Churchill Livingstone.

Fridovich I 1975. Superoxide dismutases. Ann Rev Biochem, 44: 147-159.

Hiroi S, Harada H, Nishi H, Satoh M, Nagai R, Kimura A 1999. Polymorphisms in the SOD2 and HLA-DRB1 genes are associated with nonfamilial idiopathic dilated cardiomyopathy in Japanese. Biochem Biophys Res Commu, 261: 332-339.

Huang P, Feng L, Oldham EA, Keating MJ, Plunkett W 2000. Superoxide dismutase as a target for the selective killing of cancer cells. Nature, 407: 390-395.

Li Y, Huang TT, Carlson EJ, Melov S, Ursell PC, Olson JL, Noble LJ, Yoshimura MP, Berger C, Chan PH, Wallace DC, Epstein CJ 1995. Dilated cardiomy- opathy and neonatal lethality in mutant mice lacking manganese superoxide dismutase. Nature Genet, 11: 376-381.

Lin CS, Liu CY, Sun YL, Chang LC, Chiu YT, Huang SY, Lin JH, Yang PC, Chu R, Huang MC, Mao SJ 1997. Alteration of endogenous antioxidant enzymes in naturally occurring hypertrophic cardiomyopathy. Biochem Mol Biol Int, 43(6): 1253-63.

Nishi H, Koga Y, Koyanagi T, Harada H, Imaizumi T, Toshima H, Sasazuki T, Kimura A 1995. DNA typing of HLA class II genes in Japanese patients with dilated cardiomyopathy. J Molec Cell Cardiol, 27: 2385-2392.

Novak R, Bosze Z, Matkovics B, Fachet J 1980. Gene affecting superoxide dismutase activity linked to the histocompatibility complex in $\mathrm{H}-2$ congenic mice. Science, 207: 86-87.

Zimmerman RL, Flohe U, Weser, Hartmann HJ 1973. Inhibition of lipid peroxidation in isolated inner membrane of rat liver mitochondria by superoxide dismutase. FEBS Letters, 29: 117-120. 